\title{
Hippocampal volume and depressive symptoms are linked to serum IL-18 in schizophrenia \\ OPEN
}

Paola Bossù, PhD

Fabrizio Piras, PhD Ilaria Palladino, PhD Mariangela Iorio, $\mathrm{PhD}$ Francesca Salani, PhD Antonio Ciaramella, PhD Chiara Chiapponi, $\mathrm{PhD}$ Carlo Caltagirone, MD Gianfranco Spalletta, MD

Correspondence to Dr. Bossù: p.bossu@hsantalucia.it

\section{ABSTRACT}

Objective: Since schizophrenia (SCZ) is often accompanied by hippocampal abnormalities and dysregulation of cytokine production, this study aimed to investigate the impact of the cytokine interleukin (IL)-18, whose biological system appears to be perturbed in SCZ, on brain structure and clinical severity in patients with chronic SCZ.

Methods: The serum levels of IL-18, including its free bioactive form (i.e., the cytokine fraction not bound to its specific endogenous inhibitor IL-18 binding protein), were evaluated in a case-control study involving 71 individuals with SCZ diagnosis and 29 healthy controls. All participants underwent brain MRI automatic evaluation for hippocampal volume estimation. The Positive and Negative Syndrome Scale (PANSS) was administered to measure severity of symptoms in patients with SCZ.

Results: Lower amounts of free IL-18 were related to smaller hippocampal volume measures in patients with SCZ. Furthermore, in line with a possible neuroprotective effect of the cytokine, higher levels of free IL-18 corresponded to lower subscores of PANSS depression in patients with SCZ.

Conclusions: These findings demonstrate that the levels of circulating bioactive IL-18 are related to both hippocampal volume and severity of psychopathologic symptoms in patients with SCZ, confirming the involvement of the cytokine in SCZ pathophysiology and suggesting hippocampal-dependent and neuroprotective functions of IL-18 in this clinical context. Neurol Neuroimmunol Neuroinflamm 2015;2:e111; doi: 10.1212/NXI.0000000000000111

\section{GLOSSARY}

DSM-IV-TR = Diagnostic and Statistical Manual of Mental Disorders, 4th edition, text revision; $\mathbf{H C}=$ healthy control; HPA = hypothalamic-pituitary-adrenal; HPV = hippocampal volume; ICV = intracranial volume; IL = interleukin; IL-18BP = interleukin-18 binding protein; PANSS = Positive and Negative Syndrome Scale; PANSS-PG = PANSS general psychopathology subscale; $\mathbf{S C Z}$ = schizophrenia.

Schizophrenia (SCZ) is a complex illness of unknown etiology that is characterized by heterogeneous symptoms and that impairs high brain functions, leading to a chronic and debilitating clinical outcome.

A long list of susceptibility genes, along with neurobiological alterations in brain structure, physiology, and neurochemistry, as well as immune alterations, are potential contributory factors of SCZ. ${ }^{1,2}$ In patients with both first-episode and chronic SCZ, a diminution of hippocampal volume (HPV) has been broadly reported, ${ }^{3}$ and modifications in both systemic and compartmentalized responses of cytokines have been suggested as state or trait markers ${ }^{4,5}$; however, few studies have considered the link between HPV and cytokine levels. ${ }^{6}$ In particular, interleukin (IL)-18, which is expressed and tightly regulated in brain areas (including the hippocampus ${ }^{7}$ ), tends to be elevated in the blood of patients with SCZ. ${ }^{8-11}$ Notably, the IL-18-specific inhibitor IL-18 binding protein (IL-18BP), a molecule able to modulate the activity of IL-18 by hampering its

From the Department of Clinical and Behavioral Neurology (P.B., F.P., I.P., M.I., F.S., A.C., C. Chiapponi, C. Caltagirone, G.S.), IRCCS Santa Lucia Foundation, Rome, Italy; Department of Neuroscience (C. Caltagirone), University “Tor Vergata," Rome, Italy; and Menninger Department of Psychiatry and Behavioral Sciences (G.S.), Baylor College of Medicine, Houston, TX.

Funding information and disclosures are provided at the end of the article. Go to Neurology.org/nn for full disclosure forms. The Article Processing Charge was paid by IRCCS Fondazione Santa Lucia, Rome, Italy.

This is an open access article distributed under the terms of the Creative Commons Attribution-Noncommercial No Derivative 4.0 License, which permits downloading and sharing the work provided it is properly cited. The work cannot be changed in any way or used commercially. 
binding with the receptor, ${ }^{12}$ is also increased in the blood of patients with SCZ, ${ }^{13}$ suggesting that the whole IL-18 system is perturbed in a resilient way as a possible consequence of activated peripheral and central inflammatory responses.

With the aim to better characterize this alteration in SCZ, we investigated the relationships among the serum levels of bioactive IL-18, the neuroanatomical abnormalities of the hippocampal regions, and the severity of neuropsychiatric symptoms in patients with SCZ.

METHODS Participants. One hundred patients with SCZ diagnosed according to the DSM-IV-TR were initially identified as potentially eligible for enrollment and continuously recruited between January 2010 and December 2012 from 2 outpatient clinics in Central Italy. The diagnosis of SCZ was made independently by 2 clinicians who were trained until they reached an interrater reliability level of $\kappa \geq 0.80$. A senior psychiatrist confirmed all diagnoses using the Structured Clinical Interview for DSM-IV-TR Patient Edition. ${ }^{14}$

Forty-five healthy controls (HCs), matched with the patients with SCZ in terms of age and sex, were also initially identified as potentially eligible for enrollment and continuously recruited. Exclusion criteria were as follows: (1) treatment with antiinflammatory or immunosuppressive medication; (2) presence of overt infectious disease or autoimmune disease; (3) history of alcohol or drug dependence or traumatic head injury; (4) presence of major unbalanced medical illnesses (e.g., nonstabilized diabetes, obstructive pulmonary disease or asthma, hematologic/oncologic disorders, vitamin $B_{12}$ or folate deficiency, pernicious anemia, clinically significant and unstable active gastrointestinal, renal, hepatic, endocrine, or cardiovascular disorders, and recently treated hypothyroidism); and (5) presence of vascular lesions, tumor, or clear cortical and subcortical atrophy on brain MRI. Presence, severity, and spatial location of lesions were analyzed following the Rotterdam Scan Study protocol. ${ }^{15}$

Severity of psychotic symptoms was assessed in patients with SCZ using the Positive and Negative Syndrome Scale (PANSS). ${ }^{16}$ The full scale is composed of 3 subscales: the 7 -item positive subscale, the 7-item negative subscale, and the 16-item general psychopathology subscale (PANSS-PG). Episodic memory performance was assessed using the 15-minute immediate recall of the Rey 15 -word list. ${ }^{17}$ All patients were receiving stable oral doses of one or more atypical antipsychotic drugs such as risperidone, quetiapine, and olanzapine. Antipsychotic doses were converted to equivalent doses of olanzapine.

Standard protocol approvals, registrations, and patient consents. The present study received ethical approval from the institutional review board of Santa Lucia Foundation. After receiving a full written description of the study, each participant signed the informed consent document.

MRI procedures and HPV measurement. All participants underwent the same imaging protocol, including standard clinical sequences (fluid-attenuated inversion recovery, proton density and T2-weighted) and a high-resolution T1-weighted sequence obtained using a modified driven equilibrium Fourier transform sequence (echo time/repetition time $=2.4 / 7.92 \mathrm{~ms}$, flip angle $=15^{\circ}$, voxel size $=1 \times 1 \times 1 \mathrm{~mm}^{3}$ ), in a 3T Allegra
MR imager (Siemens, Erlangen, Germany) with a standard quadrature head coil. All planar sequence acquisitions were obtained in the plane of the anterior commissure-posterior commissure line. Particular caution was taken to center participants' heads in the coil and to restrain movements with cushions. Automated labeling and quantification of HPV was performed using FreeSurfer 4.05 installed on a Centos 6.0 Linux workstation. The global FreeSurfer stream and the automated procedures for volumetric quantification of deep gray matter structures have been described elsewhere. ${ }^{18}$ Briefly, MRI volumes are registered to the Talairach space and the output images are intensity-normalized. Subsequently, the skull is stripped off through an automatic hybrid method that uses both watershed algorithms and deformable surface models. At this step, manual work is needed to edit those areas that should be corrected. Then, the output brain mask is labeled using a probabilistic atlas, ${ }^{19}$ including up to 40 structures, assigning a neuroanatomical label to each voxel based on information estimated automatically from a manually labeled training set. The Freesurfer subcortical segmentation requires these steps: (1) optimal linear transform maximizing the likelihood of the input image, given an atlas constructed from manually labeled images; (2) nonlinear transform initialized with the linear one, in order to allow the image to further deform and better match the atlas; and (3) Bayesian segmentation procedure and maximum a posteriori estimate of the labeling. The procedure provides volume (in $\mathrm{mm}^{3}$ ) of different deep gray matter assemblies, such as the thalamus, caudate nucleus, putamen, pallidum, amygdala, accumbens area, and, crucially for this study, the whole hippocampal formation (dentate gyrus, the ammonic subfields [CA1, CA2, CA3, CA4], the prosubiculum, and the subiculum).

Intracranial volume (ICV), which includes biological material such as meninges and CSF in addition to brain tissue, was calculated to correct the regional brain volume analyses. Specifically, we corrected the volume of each deep gray matter structure considered here for ICV according to the proportion method. ${ }^{20}$

Serum sampling and IL-18/IL-18BP evaluation. Blood samples were drawn from all participants in the morning, following overnight fast. After centrifugation of clotted blood, serum aliquots were obtained and stored at $-80^{\circ} \mathrm{C}$. The measurement of total IL-18, IL-18BP, and free IL-18 was performed as reported in our previous publication. ${ }^{13}$ Briefly, total IL-18 and IL-18BP levels were assessed by ELISA using a specific pair of antibodies (MBL, Nagoya, Japan) and a commercial ELISA kit (R\&D Systems, Minneapolis, MN), respectively. Both assays were performed in duplicate following the manufacturer's instructions. Limit of detection was $12.5 \mathrm{pg} / \mathrm{mL}$ for both ELISAs. Free IL-18 levels were calculated based on the law of mass action, as previously reported. ${ }^{13}$

Statistics. In order to detect statistically significant differences, comparisons between continuous variables were made using a series of 5 2-way analyses of variance, with right and left HPV and the 3 IL-18 values as the dependent variables and diagnosis and sex as the independent variables.

To determine the significance of the relationships between continuous variables, correlation analyses and Fisher $r$-to- $z$ transformation were performed. Sociodemographic, behavioral, and anatomical predictors of free IL-18 were eventually assessed by means of stepwise multiple regression analyses ( $F$ to remove $>4, p<0.05)$ using IL-18 values as dependent variables and behavioral and hippocampal values as independent variables. This multivariate statistical method permits looking for relationships without inflating the risk of a type I error. Notably, we performed 
both forward and backward stepwise regression, considering all independent variables jointly.

The level of statistical significance was defined as $p<0.05$. StatView software (SAS Institute Inc., Version 5.0.1, Cary, NC) was used for statistical analyses.

The initial sample size of our study was selected for estimating the impact of IL-18 on brain structure and symptom severity in patients with SCZ rather than for comparing the cytokine levels between patients with SCZ and HCs; the latter was described in our previous publication..$^{13}$ Hence, in order to evaluate relationships among variables, the patient sample size was calculated to show an $R^{2}$ significantly different from 0 in the multiple regression between IL-18 as dependent variable and HPV, PANSS depression factor, sex, illness duration, and Rey 15-word immediate recall score as predictors. ${ }^{21}$ In this framework, to have a medium-large Cohen effect size $f=0.20$, a statistical power of 0.8 , and a significance level $p=0.05$, a multiple regression with the 5 abovementioned parameters as regressors needs a sample of 70 participants.

RESULTS Numbers of individuals at each stage of study. A total of 100 patients with SCZ and 45 HCs were identified as potentially eligible for enrollment. Among those, 10 patients with SCZ and 7 HCs declined to participate, and a further 5 patients with SCZ and 3 HCs declined clinical and/or neuroimaging examination or blood test. Subsequently, among the individuals initially examined for eligibility, only 75 of 85 patients with SCZ and 32 of 35 $\mathrm{HCs}$ were further confirmed. In accordance with exclusion criteria, 10 patients with SCZ and 3 HCs were not eligible because of the presence of the

\begin{tabular}{|c|c|c|}
\hline \multirow[t]{2}{*}{ Demographic, biolos } & \multirow[b]{2}{*}{$\begin{array}{l}\text { Healthy } \\
\text { controls }(n=29 \text { ) }\end{array}$} & \multirow[b]{2}{*}{$\begin{array}{l}\text { Patients with } \\
\text { schizophrenia }(n=71)\end{array}$} \\
\hline & & \\
\hline Men/women, n & $20 / 9$ & $45 / 26$ \\
\hline Age, $y \pm S D$ & $41.9 \pm 11.6$ & $41.8 \pm 11.6^{\mathrm{ns}}$ \\
\hline Serum IL-18, pg/mL \pm SD & $360.6 \pm 161.7$ & $496 \pm 210 *$ \\
\hline Serum IL-18BP, $\mathrm{ng} / \mathrm{mL} \pm$ SD & $4.43 \pm 1.16$ & $10.36 \pm 3.95^{* *}$ \\
\hline Serum free IL-18, pg/mL \pm SD & $226.7 \pm 103.4$ & $208.1 \pm 84.1^{\text {ns }}$ \\
\hline MMSE score \pm SD & $29.86 \pm 0.35$ & $27.6 \pm 2.45^{* *}$ \\
\hline Disease onset age, $y \pm S D$ & - & $25.7 \pm 8.8$ \\
\hline Duration of illness, $y \pm S D$ & - & $16.1 \pm 11.26$ \\
\hline PANSS-P score \pm SD & - & $22.76 \pm 5.58$ \\
\hline PANSS-N score \pm SD & - & $20.1 \pm 7.83$ \\
\hline PANSS-PG score \pm SD & - & $47.87 \pm 13.1$ \\
\hline Olanzapine equivalents, $\mathrm{mg} / \mathrm{d} \pm \mathrm{SD}$ & - & $17.84 \pm 15.97$ \\
\hline
\end{tabular}

Abbreviations: IL $=$ interleukin; IL-18BP $=$ IL-18 binding protein; MMSE $=$ Mini-Mental State Evaluation; PANSS = Positive and Negative Syndrome Scale; PANSS-N = PANSS negative subscale; PANSS-P $=$ PANSS positive subscale; PANSS-PG $=$ PANSS general psychopathology subscale.

Analysis of variance main effect of diagnosis: $\mathrm{ns}=$ not significant; $* p<0.005 ; * * p<$ 0.0001 vs healthy controls.

There were no statistically significant diagnosis by sex interaction effects on the 3 IL-18 system molecule values. following potential confounders: major medical illnesses (4 SCZ and $1 \mathrm{HC}$ ), brain lesions (3 SCZ and $1 \mathrm{HC})$, treatment with anti-inflammatory drugs (2 SCZ), autoimmune disease (1 SCZ), and traumatic brain injury (1 HC). Finally, only 71 of 75 patients with SCZ and 29 of 32 HCs were analyzed in this study because 4 patients with SCZ and $3 \mathrm{HCs}$ were excluded due to low quality of imaging. The main demographic, biological, and clinical characteristics of all the participants analyzed in this study are summarized in table 1 . No missing data have been reported.

Group differences in serum levels of IL-18 and HPV. As shown in table 1 , and consistent with what we previously reported, ${ }^{13}$ the serum levels of free bioactive IL-18 were comparable in patients with SCZ and HCs, despite the increase of both total IL-18 and IL-18BP observed in patients with SCZ compared to HCs. Furthermore, there was no diagnosis by sex interaction for the 3 IL-18 system molecule values. No relationships were found in patients with SCZ between the equivalent doses of olanzapine and the levels of any form of IL-18 or IL-18BP.

As shown in table 2, hippocampal right and left volumes were found to be smaller in patients with SCZ than in HCs. There was no diagnosis by sex interaction for the $2 \mathrm{HPVs}$. Thus, the differences between HCs and patients with SCZ in the serum levels of IL-18 system molecules, as well as in total $\mathrm{HPV}$, were independent of sex in both groups, even though in our setting men have increased levels of total and free IL-18 (but not IL-18BP) and total hippocampal right and left volumes compared with woman in both HC and SCZ groups (data not shown, available upon request).

Relationships between serum levels of IL-18 and HPV. Since IL-18 has been previously related to hippocampal functions and patients with SCZ have HPV reduction, we considered the relationships between peripheral levels of IL-18 and HPV measures in our SCZ sample.

No relationships were observed between total IL-18 levels and hippocampal volumetric data. Higher IL-18BP levels were slightly related to smaller total volumes of both left $(r=-0.21 ; p=0.078)$ and right $(r=-0.22 ; p=0.064)$ hippocampus, with values approaching statistical significance. As shown in figure 1, lower serum amounts of free bioactive IL-18 were observable in patients with smaller volumes of both left $(r=0.29 ; p=0.014)$ and right hippocampus $(r=0.25 ; p=0.037)$. No relationships were found in HCs between the levels of any form of IL-18 or IL-18BP and HPV (data not shown).

Relationships between serum levels of bioactive IL-18 and symptom severity. Given the association between 
bioactive IL-18 serum levels and structural hippocampal abnormalities in patients with SCZ, we aimed to identify the potential impact of the circulating amounts of bioactive IL-18 on the severity of SCZ symptoms. Thus, a correlative analysis was performed between the serum levels of free IL-18 and the score of the positive, negative, and psychopathology PANSS subscales, as well as each single item of the subscales.

No relationships were observed between the levels of the 3 molecules belonging to the IL-18 system and the score of the PANSS subscales or their single items, apart from the depression factor (i.e., the sum of depression, anxiety, and feelings of guilt item scores) in the PANSS-PG subscale. In fact, as shown in figure 2, SCZ patients with increased levels of free IL-18 exhibited lower scores on the depression factor of the PANSS-PG subscale $(r=-0.30$; $p=0.011)$.

\begin{tabular}{lll|}
\hline Table 2 & Hippocampal volumetry & \\
Hemisphere & $\begin{array}{l}\text { Healthy } \\
\text { controls }\end{array}$ & $\begin{array}{l}\text { Patients with } \\
\text { schizophrenia }\end{array}$ \\
Right & $3,346 \pm 359$ & $2,957 \pm 318^{\mathrm{a}}$ \\
Left & $3,259 \pm 316$ & $2,894 \pm 327^{\mathrm{a}}$
\end{tabular}

Values are in $\mathrm{mm}^{3} \pm \mathrm{SD}$. There were no statistically significant diagnosis by sex interaction effects on the 2 hippocampal volume values.

${ }^{a}$ Analysis of variance main effect of diagnosis: $p<0.0001$ vs healthy controls.

No significant relationships were observed between symptom severity data and the levels of total IL-18 or IL-18BP.

Finally, SCZ patients with a lower depression factor score showed an increased volume of both left $(r=-0.24 ; p=0.044)$ and right hippocampus $(r=-0.32 ; p=0.007)$.

Figure 1 Association between serum levels of IL-18 and hippocampal volume in patients with schizophrenia
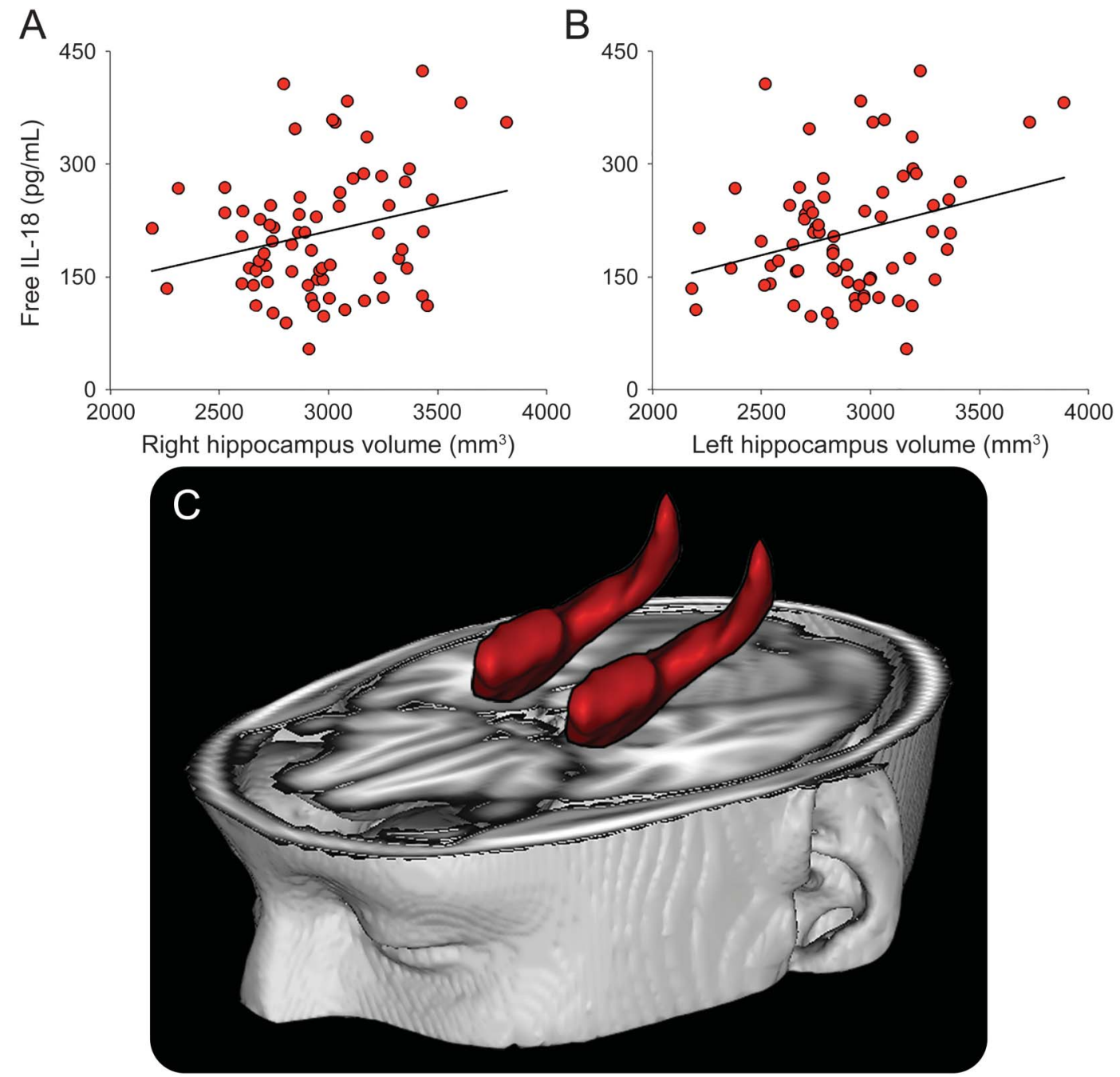

Scatter plots show a positive correlation between free bioactive interleukin (IL)-18 levels and the volume of right (A) and left (B) hippocampi, which are depicted in red and superimposed onto a standard brain (C). Solid lines in the graphs represent linear regression. 
Figure 2 Association between serum levels of IL-18 and symptom severity in patients with schizophrenia

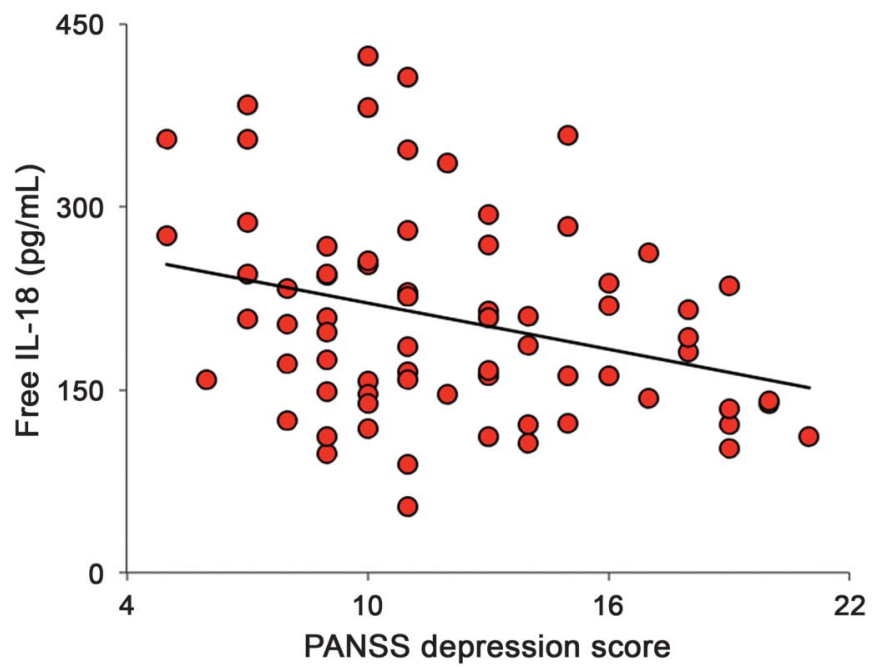

Scatter plot shows a negative correlation between free bioactive interleukin (IL)-18 levels and the depression score on the Positive and Negative Syndrome Scale (PANSS) general psychopathology subscale. Solid line represents linear regression.

Multivariate regression models of free IL-18. Two different stepwise regression analyses were performed for free IL-18 values and left and right HPV in order to avoid collinearity between HPV of the 2 hemispheres. In the overall sample of patients with SCZ, the first stepwise forward analysis including left HPV, PANSS depression factor, sex, illness duration, and Rey 15-word immediate recall score as independent variables indicated that left HPV (standard coefficient $=0.232 ; F$ to remove $=$ 4.005) and depression (standard coefficient $=$ $-0.246 ; F$ to remove $=4.508)$ were the significant predictors of free IL-18 values $(F=5.576$; $d f=2.68$; $p=0.0057)$ and explained $14.1 \%\left(R^{2}\right)$ of the variance of the dependent variable. The second stepwise forward analysis (including right HPV) indicated that depression (standard coefficient $=$ $-0.300 ; F$ to remove $=6.848$ ) was the only significant predictor of free IL-18 values $(F=$ 6.848; $\mathrm{df}=1.69 ; p=0.0109)$ and explained $9.0 \%$ $\left(R^{2}\right)$ of the variance of the dependent variable.

DISCUSSION The immune system may be of great relevance in SCZ, as inflammatory events may play a role in its pathogenesis. ${ }^{22}$ Cytokines are key mediators of the immune-brain cross-talk, and the proinflammatory cytokine IL-18 is an element of emerging importance in this scenario. ${ }^{7}$ In fact, besides being constitutively produced in microglia and neurons, its elevation in the hippocampus (a region responsive to proinflammatory cytokines and of special interest in SCZ) is a persistent consequence of inflammatory insults leading to behavioral modifications. ${ }^{23}$ Regarding its biological activity, IL-18 has many roles in the brain, ranging from proinflammatory activity and promotion of neuronal death and dysfunction in neurodegenerative conditions to optimal microglia activation and facilitation of basal synaptic transmission in hippocampal neurons. ${ }^{24,7}$

In this study, we investigated the role of IL-18 in SCZ by addressing the question of whether the circulating levels of IL-18 were related to both changes in HPV and symptom severity in patients. The measure of cytokine blood concentration is most likely relevant to brain changes because the expression levels of biological processes are often comparable between whole blood and brain, ${ }^{25}$ and cerebral function may be affected by peripheral levels of cytokines, even if these are present in low amounts. ${ }^{26}$ We found that serum levels of total IL-18 and its inhibitor were increased in our patient group compared with HCs, whereas the levels of the bioactive form of the cytokine were not significantly different between patients with SCZ and HCs. Furthermore, no association between variation of the levels in any IL-18 system molecules and antipsychotic medications was observed in our patient group. These results fully reproduce our previous data, obtained in a partially coincident sample of patients with SCZ and HCs, ${ }^{13}$ confirming the perturbation of the IL-18 system in patients with SCZ, though with no significant changes in blood levels of bioactive IL-18 and independent from antipsychotic treatment. Moreover, as broadly reported in the previous literature, ${ }^{3}$ we observed a bilateral volume reduction in hippocampal regions in patients with SCZ compared with HCs.

A new finding emerging from this study was the correlation between lower serum levels of free IL-18 and decreased HPV in patients with SCZ. This relation was evident only in patients with SCZ (not in HCs) and only when the free form of the cytokine was considered. Thus, although not different between patients with SCZ and HCs, there may be a specific role for bioactive IL-18 in modulating hippocampal structure, evidenced by the tendency of higher levels of the cytokine inhibitor IL-18BP to correlate with smaller HPV in patients with SCZ. On the whole, the bioactive form of IL-18 may play a protective role in the hippocampus of patients with SCZ, while IL-18 counteraction by IL-18BP may oppose the IL-18 effects on HPV changes. This might be possible in light of the ability of IL-18 to regulate neurogenesis, as recently suggested in an animal model with higher hippocampal IL-18 levels associated with higher numbers of new neurons and improved hippocampal function. ${ }^{27}$

Even if in disagreement with studies conducted in other neurologic disturbances in which IL-18 was supposed to promote brain damage, such as in neurodegeneration-dependent impairment of 
cognitive functions, ${ }^{28}$ these results are consistent with the growing evidence that in certain conditions IL-18 may act as a neuroprotective factor in the hippocampus. ${ }^{29,30}$ In addition, a recent publication points to IL-18 as an activator of JAK-STAT pathway through STAT3 in hippocampal cells, ${ }^{31}$ demonstrating that IL-18 can act directly on neuronal cells, possibly regulating the expression of specific genes that may be involved in synaptic plasticity and in the maintenance of adult neurogenesis. ${ }^{32}$

Another important finding of this study supporting the hypothesized protective role of bioactive IL-18 in this clinical context is the relationship between higher free IL-18 levels and lower scores on the PANSS depression factor in patients with SCZ. Regarding the impact of IL-18 on SCZ symptoms, it has been shown that serum IL-18 was higher in chronic SCZ patients with higher PANSS-PG subscores, ${ }^{10}$ whereas more recently the same authors reported that higher IL-18 levels were associated with increased cognitive performance in first-episode drugnaive patients with SCZ. ${ }^{11}$ The results of these studies are unlikely to be comparable with ours mainly because we took into consideration the bioactive form of IL-18, whereas the 2 studies measured only total IL-18, and also because of differences in total IL-18 levels, likely ascribable to the diverse state of disease progression of each study, as well as methodologic dissimilarities.

Regarding IL-18 impact specifically on depressive symptoms, there is general evidence that a link between IL-18 and depression exists, since IL-18 may participate in the regulation of the hypothalamic-pituitary-adrenal (HPA) axis activity following stress response, ${ }^{33}$ and dysregulated HPA axis activity may contribute to the onset of depression. IL-18 has been consistently described as a psychological stress-associated marker both in animal models of depression ${ }^{34}$ and in the clinical setting, where increased serum levels of IL-18 have been observed in patients with depres$\operatorname{sion}^{35}$ and high IL-18 production may increase susceptibility to depression following stress. ${ }^{36}$ On the other hand, a potential protective effect of this cytokine on depressive symptoms has been reported in stroke patients, ${ }^{37}$ highlighting the possibility that IL-18-dependent immunobiological mechanisms involved in the progression of depressive symptoms might differ depending on the type of specific depressive disturbance occurring in the context of diverse clinical conditions.

In the current study, the association between free IL-18 levels and the severity of depression may mirror the cytokine effects on the hippocampus. Indeed, there is a strong connection between hippocampal size and depression, with HPV generally reduced in patients with depression, particularly in its tail, as observed in both major depression and SCZ. ${ }^{38}$ Accordingly, in this study we observed that HPV was reduced in patients with SCZ in association with increased depressive symptom severity, in agreement with previous reports describing that reduced volume of the right and left hippocampus is related to the severity of subclinical depression symptoms. ${ }^{39}$

The results we obtained with the multiple regression analysis supported a causal relationship between IL-18 levels and psychopathological outcome and brain structure of SCZ, since depression and (left) HPV were both significant predictors of free IL-18 values in patients with SCZ. This finding corroborates the hypothesis that the modulation of the immune response induced by this cytokine may protect chronic patients from SCZ progression, possibly by attenuating the severity of depressive symptoms through mechanisms that directly or indirectly influence hippocampus integrity. This circumstance might occur despite the lack of difference in free IL-18 serum levels between patients with SCZ and $\mathrm{HCs}$, which is probably due to the suppression of this cytokine exerted by the IL-18BP elevation, which characterizes disease progression. ${ }^{13}$ This suggests that more complex mechanisms of IL-18/IL-18 receptor system modulation and signaling may exist in the brain vs the periphery, as demonstrated by recent evidence. $^{31}$

However, more research is certainly warranted to determine how IL-18 activity, hippocampal size, and symptom progression are linked together, which of these factors are driving and which are driven, and, ultimately, which molecular mechanisms underlie the IL-18 contribute to SCZ pathogenesis and/or progression. Furthermore, the present study may be limited by the relatively small sample size, and larger studies should be performed to validate our findings.

Despite its limitations, our study reports the psychopathological and brain structural correlates of circulating bioactive IL-18 in SCZ, confirming a significant interaction of this cytokine with the disturbance. In addition, our study prompts reconsideration of the multifaceted role of IL-18 in brain disorders and its suitability as a target in the development of anti-inflammatory therapeutic intervention against SCZ, a fast-growing area of research.

\section{AUTHOR CONTRIBUTIONS}

P.B. and G.S. planned and coordinated the study. C. Caltagirone and G.S. directed patient selection and clinical evaluations. I.P., F.S., and A.C. prepared the blood samples, executed ELISA, and interpreted the cytokine data. F.P., M.I. and C. Chiapponi performed the neuroimaging study, including data analysis and acquisition and statistical analyses of the results. All authors made significant contributions to the interpretation and discussion of the results. P.B. wrote the manuscript, and F.P. and G.S. critically revised it. All the authors made contributions in writing and discussing the manuscript and read and approved the final version of the manuscript. 


\section{ACKNOWLEDGMENT}

The authors thank all the patients and volunteers who provided samples for the study and Federica Bizzoni for providing outstanding technical assistance.

\section{STUDY FUNDING}

This study was supported in part by Italian Ministry of Health, RC-12-13-14 (P.B. and G.S.) and in part by the ERANET-NEURON 2010 grant.

\section{DISCLOSURE}

P. Bossù received research support from the Italian Ministry of Health. F. Piras I. Palladino, M. Iorio, F. Salani, A. Ciaramella, and C. Chiapponi report no disclosures. C. Caltagirone received travel honoraria from Pyramal Limited, is on the editorial board for Neurological Sciences, and received research support from Italian Ministry of Health, Fondazione Santa Lucia. G. Spalletta has consulted for and is on the speakers' bureau for Novartis and FB Health; received travel funding from Servier; and received research support from Italian Ministry of Health. Go to Neurology.org/nn for full disclosure forms.

Received August 19, 2014. Accepted in final form March 24, 2015.

\section{REFERENCES}

1. Sawa A, Snyder SH. Schizophrenia: diverse approaches to a complex disease. Science 2002;296:692-695.

2. Müller N, Schwarz MJ. Immune system and schizophrenia. Curr Immunol Rev 2010;6:213-220.

3. Adriano F, Caltagirone C, Spalletta G. Hippocampal volume reduction in first-episode and chronic schizophrenia: a review and meta-analysis. Neuroscientist 2012;18:180-200.

4. Potvin S, Stip E, Sepehry AA, Gendron A, Bah R, Kouassi E. Inflammatory cytokine alterations in schizophrenia: a systematic quantitative review. Biol Psychiatry 2007;63:801-818.

5. Miller BJ, Buckley P, Seabolt W, Mellor A, Kirkpatrick B. Meta-analysis of cytokine alterations in schizophrenia: clinical status and antipsychotic effects. Biol Psychiatry 2011;70:663-671.

6. Frodl T, Amico F. Is there an association between peripheral immune markers and structural/functional neuroimaging findings? Prog Neuropsychopharmacol Biol Psychiatry 2014; 48:295-303.

7. Alboni S, Cervia D, Sugama S, Conti B. Interleukin 18 in the CNS. J Neuroinflammation 2010;7:9.

8. Tanaka KF, Shintani F, Fujii Y, Yagi G, Asai M. Serum interleukin-18 levels are elevated in schizophrenia. Psychiatry Res 2000;96:75-80.

9. Reale M, Patruno A, De Lutiis MA, et al. Dysregulation of chemo-cytokine production in schizophrenic patients versus healthy controls. BMC Neurosci 2011;12:13.

10. Xiu MH, Chen da C, Wang D, et al. Elevated interleukin18 serum levels in chronic schizophrenia: association with psychopathology. J Psychiatr Res 2012;46:1093-1098.

11. Zhang XY, Tang W, Xiu MH, et al. Interleukin 18 and cognitive impairment in first episode and drug naive schizophrenia versus healthy controls. Brain Behav Immun 2013;32:105-111.

12. Novick D, Kim SH, Fantuzzi G, Reznikov LL, Dinarello CA, Rubinstein M. Interleukin-18 binding protein: a novel modulator of the Th1 cytokine response. Immunity 1999;10:127-136.

13. Palladino I, Salani F, Ciaramella A, et al. Elevated levels of circulating IL-18BP and perturbed regulation of IL-18 in schizophrenia. J Neuroinflammation 2012;9:206.
14. First MB, Spitzer RL, Gibbon M, Williams JBW. Structured Clinical Interview for DSM-IV-TR Axis I Disorders, Research Version, Patient Edition. (SCID-I/P). New York: New York State Psychiatric Institute; 2002.

15. Ikram MA, van der Lugt A, Niessen WJ, et al. The Rotterdam Scan Study: design and update up to 2012. Eur J Epidemiol 2011;26:811-824.

16. Kay SR, Fiszbein A, Opler LA. The positive and negative syndrome scale (PANSS) for schizophrenia. Schizophr Bull 1987;13:261-276.

17. Carlesimo GA, Caltagirone C, Gainotti G; Group for the Standardization of the Mental Deterioration Battery. The Mental Deterioration Battery: normative data, diagnostic reliability and qualitative analyses of cognitive impairment. Eur Neurol 1996;36:378-384.

18. Fischl B, Salat DH, Busa E, et al. Whole brain segmentation: automated labeling of neuroanatomical structures in the human brain. Neuron 2002;33:341-355.

19. Destrieux C, Fischl B, Dale A, Halgren E. Automatic parcellation of human cortical gyri and sulci using standard anatomical nomenclature. Neuroimage 2010;53:1-15.

20. Sanfilipo MP, Benedict RHB, Zivadinov R, Bakshi R. Correction for intracranial volume in analysis of whole brain atrophy in multiple sclerosis: the proportion vs. residual method. Neuroimage 2004;22:1732-1743.

21. Cohen J, Cohen P, West SG, Aiken LS. Applied Multiple Regression/Correlation Analysis for the Behavioral Sciences, 3rd ed. Mahwah, NJ: Lawrence Erlbaum Associates; 2003.

22. Meyer U, Schwarz MJ, Müller N. Inflammatory processes in schizophrenia: a promising neuroimmunological target for the treatment of negative/cognitive symptoms and beyond. Pharmacol Ther 2011;132:96-110.

23. Bossù $\mathrm{P}$, Cutuli $\mathrm{D}$, Palladino $\mathrm{I}$, et al. A single intraperitoneal injection of endotoxin in rats induces long-lasting modifications in behavior and brain protein levels of TNF- $\alpha$ and IL-18. J Neuroinflammation 2012;9:101.

24. Bossù $\mathrm{P}$, Ciaramella $\mathrm{A}$, Salani $\mathrm{F}$, et al. Interleukin-18, from neuroinflammation to Alzheimer's disease, Curr Pharm Des 2010;16:4213-4224.

25. Sullivan PF, Fan C, Perou CM. Evaluating the comparability of gene expression in blood and brain. Am J Med Genet B Neuropsychiatr Genet 2006;141B:261-268.

26. Pollmächer T, Haack M, Schuld A, Reichenberg A, Yirmiya R. Low levels of circulating inflammatory cytokines - do they affect human brain functions? Brain Behav Immun 2002;16:525-532.

27. Speisman RB, Kumar A, Rani A, Foster TC, Ormerod BK. Daily exercise improves memory, stimulates hippocampal neurogenesis and modulates immune and neuroimmune cytokines in aging rats. Brain Behav Immun 2013;28: 25-43.

28. Bossù $\mathrm{P}$, Ciaramella $\mathrm{A}$, Salani $\mathrm{F}$, et al. Interleukin-18 produced by peripheral blood cells is increased in Alzheimer's disease and correlates with cognitive impairment. Brain Behav Immun 2008;22:487-492.

29. Ryu HJ, Kim JE, Kim MJ, et al. The protective effects of interleukin-18 and interferon- $\gamma$ on neuronal damages in the rat hippocampus following status epilepticus. Neuroscience 2010;170:711-721.

30. Zhang XM, Duan RS, Chen Z, et al. IL-18 deficiency aggravates kainic acid-induced hippocampal neurodegeneration in C57BL/6 mice due to an overcompensation by IL-12. Exp Neurol 2007;205:64-73. 
31. Alboni S, Montanari C, Benatti C, et al. Interleukin 18 activates MAPKs and STAT3 but not NF-кB in hippocampal HT-22 cells. Brain Behav Immun 2014;40:85-94.

32. Murase S, Kim E, Lin L, Hoffman DA, McKay RD. Loss of signal transducer and activator of transcription 3 (STAT3) signaling during elevated activity causes vulnerability in hippocampal neurons. J Neurosci 2012;32:15511-15520.

33. Sugama $S$, Conti B. Interleukin-18 and stress. Brain Res Rev 2008;58:85-95.

34. Kroes RA, Panksepp J, Burgdorf J, Otto NJ, Moskal JR. Modeling depression: social dominance-submission gene expression patterns in rat neocortex. Neuroscience 2006; 137:37-49.

35. Merendino RA, Di Rosa AE, Di Pasquale G, et al. Interleukin-18 and CD30 serum levels in patients with moderatesevere depression. Mediators Inflamm 2002;11:265-267.
36. Haastrup E, Bukh JD, Bock C, et al. Promoter variants in IL18 are associated with onset of depression in patients previously exposed to stressful-life events. J Affect Disord 2012;136:134-138.

37. Bossù $\mathrm{P}$, Salani F, Cacciari $\mathrm{C}$, et al. Disease outcome, alexithymia and depression are differently associated with serum IL-18 levels in acute stroke. Curr Neurovasc Res 2009;6:163-170.

38. Maller JJ, Daskalakis ZJ, Thomson RHS, Daigle M, Barr MS, Fitzgerald PB. Hippocampal volumetrics in treatment-resistant depression and schizophrenia: the devil's in de-tail. Hippocampus 2012;22:9-16.

39. Spalletta G, Piras F, Caltagirone C, Fagioli S. Hippocampal multimodal structural changes and subclinical depression in healthy individuals. J Affect Disord 2014;152-154: 105-112. 


\title{
Neurology \\ Neuroimmunology \& Neuroinflammation
}

\author{
Hippocampal volume and depressive symptoms are linked to serum IL-18 in \\ schizophrenia \\ Paola Bossù, Fabrizio Piras, Ilaria Palladino, et al. \\ Neurol Neuroimmunol Neuroinflamm 2015;2; \\ DOI 10.1212/NXI.0000000000000111
}

This information is current as of May 7, 2015

\author{
Updated Information \& \\ including high resolution figures, can be found at: \\ Services \\ http://nn.neurology.org/content/2/4/e111.full.html \\ References \\ This article cites 37 articles, 2 of which you can access for free at: \\ http://nn.neurology.org/content/2/4/e111.full.html\#\#ref-list-1 \\ Subspecialty Collections \\ This article, along with others on similar topics, appears in the \\ following collection(s): \\ All Immunology \\ http://nn.neurology.org//cgi/collection/all_immunology \\ MRI \\ http://nn.neurology.org//cgi/collection/mri \\ Psychosis \\ http://nn.neurology.org//cgi/collection/psychosis \\ Permissions \& Licensing \\ Information about reproducing this article in parts (figures,tables) or in \\ its entirety can be found online at: \\ http://nn.neurology.org/misc/about.xhtml\#permissions \\ Reprints \\ Information about ordering reprints can be found online: \\ http://nn.neurology.org/misc/addir.xhtml\#reprintsus
}

Neurol Neuroimmunol Neuroinflamm is an official journal of the American Academy of Neurology.

Published since April 2014, it is an open-access, online-only, continuous publication journal. Copyright $(\subseteq$ 2015 American Academy of Neurology. All rights reserved. Online ISSN: 2332-7812.

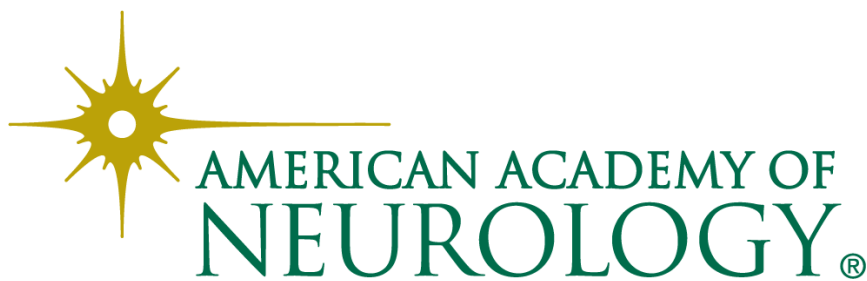

